\title{
"No vine para acompañarme, vine para trabajar." \\ O cómo las mujeres \\ centroamericanas organizan sus hogares transnacionales en \\ Belice City
}

Nanneke Winters

Institute of Development Policy and Management (IOB), University of Antwerp - Belgium. Correo electrónico:

$\underline{\text { Nanneke.Winters@ua.ac.be }}$

Recibido: noviembre de 2009 / Aceptado: noviembre de 2009

A PESAR DEL CRECIENTE INTERÉS QUE HA SUSCITADO EL ESTUdIO DE LOS PROCESOS $\mathrm{DE}$ migración internacional desde una perspectiva de género, la mayoría de los estudios solamente describe la migración de hombres y mujeres por separado sin tener en cuenta sus interacciones, o reflexiona sobre la cuestión del empoderamiento de la mujer a través de la migración. Sin embargo, la transformación de familias, hogares y comunidades derivada de la migración es mucho más compleja y requiere de un enfoque relacional más detallado y profundo. En este artículo intento contribuir al estudio de este tema a través del análisis de un componente fundamental de la migración internacional, que es la organización de hogares transnacionales. Con objeto de poder aplicar una perspectiva de género en el análisis de la organización de hogares transnacionales, aplicaré el marco teórico gendered geographies of power desarrollado por Sarah Mahler y Patricia Pessar. El presente estudio está basado en un trabajo de campo sobre las diferentes estrategias que utilizan las mujeres (y hombres) centroamericanos que emigran a Belice City para 'salir adelante'. Sus historias muestran cómo las mujeres migrantes intentan utilizar su condición de mujer transnacional a su favor. A partir de sus experiencias y perspectivas, argumento que el discurso mundial de la migración internacional necesita de un replanteamiento que tome en cuenta la interacción de hombres y mujeres y las geometrías de poder en que se encuentran los migrantes.

Palabras clave: migración / hogares transnacionales / género / Centroamérica / Belice 


\section{Introducción}

Según sus habitantes, Belice City ya no se ve como antes. En el mosaico cultural que es Belice, la vieja capital siempre ha sido de los beliceños criollos ${ }^{1}$, pero en los últimos años más y más Spanish people ${ }^{2}$ vinieron. En poco tiempo, estos migrantes centroamericanos han cambiado la cara de la ciudad. Sobre todo las mujeres migrantes llegaron a ser más visibles. Estas latinas ${ }^{3}$ ponen sus carritos de venta en puntos estratégicos para vender comida rápida a empresarios, estudiantes y obreros de todo color. También son ellas las que todos los domingos llenan el parque más popular de la ciudad para vender alimentos a las familias beliceñas que hacen su paseo semanal. Otras trabajan en el mercado, dentro de las casas y en los bares. La mayoría de estas latinas vino a Belice City con el objetivo de, como dicen ellas, 'salir adelante'. Para lograrlo, ellas utilizan el escenario transnacional que se ha originado con su migración.

Después de mucho tiempo en que la migración era sólo algo masculino, últimamente ha surgido numerosa literatura en relación a mujeres como estas latinas, migrantes que dejan su hogar para buscar una vida mejor en otro país. Pero a pesar de este creciente interés hacia el papel del género en la migración internacional, el género como enfoque fundamental de análisis en estudios de migración sigue siendo la excepción (Mahler \& Pessar, 2006). En primer lugar, la mayoría de estos estudios solamente reflexiona sobre la vulnerabilidad de las mujeres migrantes, o, pasando a otro extremo, sobre la cuestión del empoderamiento de la mujer a través de la migración (Hirsch, 1999, p. 1333). En segundo lugar, muchos de estos estudios solamente describen las experiencias migratorias de hombres y mujeres por separado sin tener en cuenta sus interacciones. Sin embargo, la transformación de individuos, familias y hogares, derivada de la migración, es mucho más compleja y requiere de un enfoque que relacione simultáneamente diferente actores y procesos. En este artículo pretendo contribuir al estudio de este tema a través del análisis de un componente fundamental de la migración internacional, como es la organización de hogares transnacionales (Chant, 1998; Escrivá, 2000; Gardner \& Grillo, 2002). Con el concepto de organización de hogares transnacionales me refiero a las maneras en que los miembros de una red doméstica (basada en la familia e internacionalmente dispersa en diferentes lugares; ver Stack, 1974, p. 101) deciden y realizan las tareas de producción y reproducción, principalmente la generación de ingresos y el cuidado y socialización de los miembros (González de la Rocha, 2001; Oso Casas, 2008).

Durante dos estancias de seis meses en Belice City entre 2004 y 2006 conocí la dinámica de la migración centroamericana por primera vez. Este artículo está basado en un trabajo de campo siguiente de casi tres meses, en 2007, sobre las experiencias migratorias de latinas en Belice City. Realicé 60 entrevistas con cinco hombres y 35 mujeres migrantes entre 16 y 35 años de edad. La edad promedio era de 24 años. Aunque son pocos los hombres que he podido incluir en la investigación, sus puntos de vista eran cruciales para llegar a una mejor comprensión del tema. Los migrantes, con experiencias muy diversas en términos de vivienda, familia, educación y trabajo, provenían de Guatemala, El Salvador y Nicaragua, pero la mayoría era de Honduras ${ }^{4}$; porcentajes que aproximadamente reflejan la situación actual en Belice. 


\section{Transnacionalismo, género y organización de hogares transnacionales}

Debido a su impacto enorme, en las últimas décadas la migración internacional ha recibido bastante atención de gobiernos, organizaciones internacionales y académicos. Aunque los esfuerzos para teorizar el fenómeno no han culminado en una sola teoría de la migración internacional (Arango, 2000), han contribuido a una mejor comprensión de la misma. Seguidamente, antes de pasar a la discusión sobre los resultados del trabajo de campo, comentaré algunas contribuciones teóricas eminentes que se originaron en los estudios de transnacionalismo, género y organización de hogares transnacionales.

\subsection{Transnacionalismo}

En diferentes estudios prominentes sobre migración internacional se destaca que los migrantes no necesariamente rompen los vínculos con sus hogares en el país de origen. Al contrario, la mayoría de los migrantes mantienen y fortalecen estos vínculos para organizar su migración, para darles un futuro mejor a sus familiares y para asegurarse a si mismos la posibilidad del retorno (Evergeti \& Zontini, 2006, p. 1031). En los estudios sobre migración internacional, la perspectiva transnacional ha sido muy útil para analizar estos vínculos que causan que los migrantes tengan un pie en su país de origen y un pie en su país de destino (Glick Schiller, Basch \& Blanc-Szanton, 1992; Vertovec, 2004). El concepto de transnacionalismo se refiere a los procesos económicos, políticos, culturales y sociales de vinculación que los migrantes establecen entre su país de origen y su país de destino (Glick Schiller et al., 1992, pp. 26-27). Analizar los posibles vínculos que comprometen a los migrantes a dos (o más) países al mismo tiempo es una manera útil para interpretar cómo organizan sus vidas.

A la vez, la perspectiva transnacional provee una crítica a los estudios de migración que están basados en categorías fijas y 'objetivas' (Bailey, 2001, pp. 415-416). Ejemplos de estas categorías son: el tipo de migrante, la distancia y duración de la migración, la movilidad voluntaria o involuntaria, etcétera. Bailey argumenta que la importancia del transnacionalismo está en su carácter interdisciplinario, obtenido conectando "ethnographic evidence on daily lives to broader accounts of the changing nature of economic and cultural systems" (2001, p. 420).

Así, el transnacionalismo parece ser capaz de abarcar la complejidad de la migración internacional. Sin embargo, esto no quiere decir que cada migrante internacional está metido en procesos transnacionales (Vertovec, 2004, p. 2), o que migrar internacionalmente es la única manera en la que se puede participar en el transnacionalismo (2004, pp. 8 y 20). Las personas que no migran se pueden ver relacionadas con actividades transnacionales porque éstas tienen un poder transformativo que alcanza mucho más allá de las vidas de los migrantes.

El gran atractivo del transnacionalismo deriva de su potencial para proveer una nueva manera "to think about the connections between "here' and 'there"" (Waldinger \& Fitzgerald, 2004, p. 1177). Sin embargo, la perspectiva transnacional ha sido criticada también. Estas críticas incluyen la pregunta de si la migración contemporánea es realmente diferente 
a procesos de migración del pasado y por eso necesita una nueva perspectiva, y si el transnacionalismo es realmente diferente a otros marcos que intentan explicar la migración internacional (Kivisto, 2001). Además, existe una falta de claridad sobre la definición del transnacionalismo y la discusión sobre su alcance e importancia (Levitt, DeWind \& Vertovec, 2003, p. 565). Pero a pesar del debate sobre la novedad y viabilidad del transnacionalismo, la mayoría de los académicos estaría de acuerdo en que la migración internacional -en gran parte por cambios en el transporte y la comunicación- se ve diferente hoy en día y que por eso es útil avanzar la teoría del transnacionalismo (Portes, Guarnizo \& Landolt, 1999; Pries, 1999). Una manera para avanzar la perspectiva transnacional y evaluar su potencial es aplicándola a procesos menos estudiados, como es el papel del género en la migración SurSur (por ejemplo, en Centroamérica, ver Morales \& Castro, 2002).

\subsection{Género}

Los estudios de género han mostrado que no se puede analizar la migración internacional sin tomar en cuenta normas y prácticas de género. Son éstas las que son clave para la organización de cualquier tipo de migración (Hondagneu-Sotelo, 1994). Este reconocimiento de la importancia del género empezó en los años 70 y 80, cuando se pidió más atención para el papel de las mujeres en la migración (Pessar \& Mahler, 2003, p. 814). Éstas pasaron de no existir en los estudios de migración a convertirse en agentes principales en éstos. Lamentablemente, muchas veces solamente se añadió la variable sexo al análisis existente, o se puso tanta atención a las mujeres migrantes que casi se olvidó el papel de los hombres. Vale la pena enfatizar que no son estudios de hombres y estudios de mujeres migrantes lo que hace falta sino estudios que relacionen las experiencias de los dos de modo que arrojen luz sobre cómo el género organiza la migración (Donato, Gabaccia, Holdaway, Manalansan \& Pessar, 2006).

El hecho de que el género organiza la movilidad humana significa que las normas y prácticas del género juegan un papel importante en formar la migración y viceversa (Portes, 1997, p. 816) a través de su interacción continua con procesos políticos, económicos, sociales y culturales. Esta interacción se pierde cuando uno solamente se enfoca en las mujeres, frecuentemente perdiéndose en estereotipos de mujeres vulnerables o liberadas. Los estudios de experiencias migratorias de mujeres son "useful first steps toward gender analysis, but ... they are too limited in what they tell us about gender as a way of structuring power in all human relationships, including those among people on the move" (Donato et al. 2006, pp. 5-6).

\section{Gendered geographies of power}

La falta de gender sensitivity (Grieco \& Boyd, 1998) y la abundancia de estereotipos, clichés y simplificaciones (Ramírez, García Domínguez \& Míguez Morais, 2005) en estudios y teorías sobre la migración internacional han inspirado a Mahler y Pessar (2001), entre otras personas, para darle un lugar central al género. Estas autoras desarrollaron el marco teórico gendered geographies of power, definido como: 
a theoretical approach for how to conceptualize and study gendered identities and relations when conducted and negotiated across international borders, as they relate to multiple axes of difference, and as they operate along and across many sociospatial scales - from the body to the globe (Mahler \& Pessar, 2006, p. 42).

El marco teórico gendered geographies of power tiene tres componentes: espacios geográficos, sitio social y geometría del poder. El primer componente, espacios geográficos, se refiere a la manera en que las normas y prácticas del género se forman dentro y entre diferente espacios (políticos, sociales, culturales, etcétera). El segundo componente, sitio social, se refiere a la posición (indeterminada) de personas y grupos en las jerarquías de género, clase, etnicidad, etcétera. El tercer componente, geometría del poder, se refiere a la agencia que personas y grupos pueden utilizar, sobre todo dependiendo de su acceso a recursos y de sus posibilidades para negociar este acceso. En el contexto de la migración, este último componente particularmente se refiere al acceso y agencia respecto a la movilidad (Pessar \& Mahler, 2003, p. 817), lo que Massey (1993, p. 61) llama la power-geometry of time-space compression.

El marco teórico gendered geographies of power provee una manera para estudiar la migración internacional desde la perspectiva del transnacionalismo y el género. A lo largo de este artículo utilizaré este marco para interpretar las vidas de las latinas en Belice City, enfocándome en los diferentes espacios y dimensiones que están relacionados a su migración, su posición en las diferentes jerarquías de poder y su agencia; temas que se manifiestan a través de una organización particular de sus hogares transnacionales.

\subsection{Organización de hogares transnacionales}

Diferentes autores han argumentado que el hogar doméstico (al que llamaré hogar en la discusión siguiente) es un sitio de análisis útil para estudios de migración (Chaney, 1993, p. 103; Chant \& Craske, 2003, pp. 229-230; Morales \& Castro, 2002, p. 140; Ribas, 2001, p. 89; Stephen, 1993, p. 77). Se puede ver el hogar como una institución social (Chant, 1998, p. 18; Posso Quiceno, 2007, p. 7) en la que convergen procesos estructurales y agencia individual (Escrivá, 2000, p 331; González de la Rocha, 2001, p. 74; Oliveira, 1991, pp. 110-111). Así, el hogar parece ser un sitio lógico para estudiar la migración, que tiene sus raíces tanto en procesos políticos, económicos, sociales y culturales, como en las imaginaciones, actitudes $\mathrm{y}$ actividades de individuos y sus familias.

Acknowledging the household as the most immediate institution affecting men and women's power, behaviour and identities, it is not surprising that research undertaken from a gender and/or feminist perspective has begun to consider the analysis of this institution, in all its diverse guises, as fundamental to gaining a more nuanced theoretical grasp of gender-selective migration, most notably under the auspices of a 'household strategies approach' to population mobility (Chant, 1998, p. 9).

Sin embargo, no es fácil llegar a una comprensión del hogar que coincida con la realidad, es decir una comprensión que permita analizar las relaciones de poder que rodean el hogar 
(Carr, 2005, p. 81). Para poder utilizar el hogar como unidad analítica, por lo menos dos interpretaciones comunes del hogar necesitan replanteamiento. En primer lugar, no se puede ver el hogar como un sitio estable con una cantidad fija de miembros y basado en un solo lugar. Esta definición simplista del hogar ha sido rebatida por ser basada en una noción occidental de familias nucleares que no toma en cuenta composiciones dinámicas que son más comunes en muchos países en desarrollo. "Households are not 'natural' units with fixed forms and meanings across space and through time but are socially constructed and inherently variable" (Chant, 1998, p. 7; ver también Carr, 2005). Además, particularmente la migración y la perspectiva transnacional incitan a "reconsider our understanding of households and families based on the idea of co-residency and physical unity and to take into account the possibility of spatial separation" (Evergeti \& Zontini, 2006, p. 1032). Cuando un miembro de un hogar migra, no quiere decir que él o ella ya no forma parte de este hogar, sino que las actividades básicas de producción y reproducción (Carr, 2005, p. 72, Oso Casas 2008) se ven dispersas. Reconocer la composición dinámica y la multilocalidad del hogar hace posible tomar los hogares como unidades analíticas en los estudios de migración. En este contexto, podría ser útil hablar de una red doméstica basada en la familia e internacionalmente dispersa en diferentes lugares (Stack, 1974, p. 101). Así se puede identificar hogares separados e investigarlos como parte de redes internacionales que les dan un carácter transnacional.

En segundo lugar, la interpretación común del hogar como un sitio armonioso, basado en la igualdad de sus miembros y la lucha para objetivos colectivos ya no es sostenible. Académicas feministas en particular (Chant, 1998, p. 8) han señalado los power plays que ocurren dentro de los hogares. Estos power plays están basados primariamente en diferencias de género y generación (González de la Rocha, 2001, p. 76; Molyneux, 2002, p. 183), determinan la organización de tareas productivas y reproductivas, y pueden hasta contribuir a niveles desiguales de bienestar dentro del hogar (Chant, 2007, p. 113). A pesar de que cierto nivel de cohesión y solidaridad es necesario para poder funcionar como hogar (González de la Rocha, 1994, pp. 13-14; Pribilsky, 2004, p. 316), los diferentes miembros del hogar tienen diferentes intereses y diferentes niveles de poder para tomar decisiones. Así que el hogar es un sitio crucial para entender la movilidad (Chant \& Craske, 2003, p. 229; de Haan, 2006, p. 15).

\section{Migrantes centroamericanos en Belice City}

Belice, un pequeño enclave caribeño de sólo 23.000 metros cuadrados entre México, Guatemala y el Mar Caribe, tiene una población de no más de 300,000 personas, que mayoritariamente consiste en mestizos, criollos, mayas y garífunas (Central Intelligence Agency [CIA], 2009). A pesar de su posición geográfica, Belice siempre ha estado un poco aislado de los procesos económicos, políticos y socioculturales de Centroamérica (Morales Gamboa, 2007, pp. 174-178). Pero en cuanto a la migración internacional dentro de la región, Belice juega un papel importante. Desde la década de los ochenta, ha sido el país centroamericano relativamente más afectado por este tipo de migración. El porcentaje de inmigrantes era casi $15 \%$ en 2000 , cerca de la mitad mujeres, con más de $75 \%$ proviniendo de la región centroamericana. Antes, la mayoría de los migrantes vino a trabajar en la agricultura, pero en los últimos años la vieja capital Belice City tiene mayor atractivo 
(Organización Internacional para las Migraciones / Sistema de Información Estadística sobre las Migraciones en Centroamérica [OIM/SIEMCA], 2004). Es llamativo que a pesar de que la presencia de migrantes centroamericanos ha contribuido a crear tensiones entre ellos y los beliceños, se describe el proceso de la inmigración centroamericana como algo relativamente pacífico.

Las latinas que llegan aquí para buscar una vida mejor tienen experiencias muy diversas en términos de vivienda, familia, educación y trabajo en sus países de origen. Pero a través de su migración a Belice City, sus experiencias convergen de dos maneras: casi todas las mujeres trabajan, y la mayoría vive junto con un hombre que es su pareja. Abajo abordaré estos tres temas -migración, trabajo y la organización de hogares transnacionales- que se reúnen en el concepto de 'salir adelante'.

\subsection{El acceso a la migración}

Para las latinas es relativamente fácil llegar a Belice ${ }^{5}$. El viaje es visto como algo barato, corto y cómodo. Además, los centroamericanos no necesitan visa para entrar al país ${ }^{6}$. La accesibilidad de Belice se ve potenciada por la dinámica de la toma de decisiones dentro de los hogares familiares de las latinas en su país de origen. Ellas describen la decisión de su migración como poco conflictiva. En una situación en que los sueldos de los hombres ya no llegan a cubrir los gastos de la casa (ver Chant, 2007, p. 333 y Sassen, 2002, p. 257) y la búsqueda de trabajo es cada vez más difícil, las expectativas de la activa contribución de las latinas a los ingresos del hogar familiar parecen ser más fuertes que las normas 'tradicionales' que mantienen a las mujeres en casa (Chant \& Craske 2003, p. 167). Tanto su deseo de ayudar a su familia como sus ganas de aventurarse contribuyen a la decisión de migrar a un lugar cercano y bastante familiar donde se puede ganar más dinero.

El acceso fácil de las latinas a la migración hacia Belice se puede situar en los espacios políticos, económicos, culturales y sociales que las ponen en una posición ventajosa respecto a una posible migración. Desde esta posición, ellas pueden ejercer la agencia necesaria para participar en procesos migratorios. El tráfico libre de personas en Centroamérica, la percepción común de las mejores oportunidades económicas en Belice y las responsabilidades familiares de las mujeres facilitan el acceso a la migración para aquellas que sueñan con 'salir adelante'.

\subsection{Organizar cómo 'salir adelante' en los hogares transnacionales}

A pesar de las muchas diferentes interpretaciones que existen de la migración internacional, hay consenso sobre el hecho de que la gente decide migrar para mejorar su vida (Zoomers, 2006). Este mejoramiento tiene un fuerte aspecto económico, lo cual también surgió en mis conversaciones con las latinas. Dicen que en Belice hay trabajo, 'aquí se gana'. El objetivo de su migración es ganar dinero para buscar una vida mejor, sacar la familia adelante, progresar. En otras palabras, las latinas han venido a Belice City para 'salir adelante'.

Sin embargo, a pesar del énfasis en el aspecto económico, hay que tomar en cuenta que los motivos para migrar son muy complejos y dinámicos (Hondagneu-Sotelo, 1994, p. 69; Jolly 
\& Reeves, 2005, p. 8). Cuando se analiza el concepto de 'salir adelante' dentro de la red doméstica de las latinas, su organización revela las emociones que están relacionadas a la migración y muestra que se trata de mucho más que solamente consideraciones económicas. Aclararé brevemente la red doméstica. Consiste de por lo menos dos hogares: el hogar familiar en el país de origen donde vivían las latinas antes de venir a Belice (al que llamaré hogar familiar) y el nuevo hogar que ellas están construyendo en Belice City (al que llamaré nuevo hogar). Los hogares familiares de las latinas son muy diversos, pero la gran mayoría vivía con sus padres, hermanos y otros familiares. En sus nuevos hogares en Belize City, el número de latinas que vive con su familia ha disminuido significativamente ${ }^{7}$ y el número de éstas que vive con una pareja se ha doblado hasta llegar a la mitad de ellas. Algunas latinas han dejado hijos en el país de origen. Generalmente, son mujeres de la familia las que cuiden de ellos allá. En Belice City, casi la mitad de las latinas vive con sus hijos. Aquí las latinas tienen que pagar para que los cuiden mujeres que no son de la familia.

A pesar de que las latinas siempre mencionan ayudar a su familia en el país de origen como un motivo fundamental para migrar -lo cual coincide con la literatura (ver Tamagno, 2003)en su explicación del concepto 'salir adelante' sus propios proyectos tienen un lugar más significativo. Sin embargo, estos proyectos casi siempre están orientados hacia su país de origen. ${ }^{8}$ Más de la mitad de las latinas está trabajando para obtener su propia casa y su propia empresa allá. Otras metas son conseguir un mejor trabajo, mandar a sus hijos al colegio, tener más cosas, ahorrar y (en este orden) ayudar a su familia. El hecho de que las diferentes metas dentro del concepto 'salir adelante' estén orientadas hacia lograr cierta independencia en el país de origen tiene consecuencias para las estrategias que las latinas utilizan para lograrlas. Como explicaré más adelante, los dos hogares de su red doméstica juegan su propio papel en estas estrategias.

\subsection{Expectativas familiares y relaciones estratégicas}

Las latinas mandan remesas para ayudar a su familia y así cumplir con sus responsabilidades familiares, pero también para asegurarse una justificación de trabajar afuera y estar lejos de casa. Además, aunque no se sabe si ellas realmente van a regresar, casi todas las latinas ven su futuro en su país de origen. El hecho de que gran parte de su vida en Belice esté orientada hacia su país de origen, hace que mantener buenos vínculos con sus familiares allá se convierta en algo muy importante para ellas. Esta importancia no sólo se debe a que quieran mucho a sus familiares o a que ellos estén cuidando, en algunas ocasiones, de sus hijos sino, también a que cuando regresen a su país de origen necesitarán de su aprobación para poder vivir bien.

El problema es que muchas latinas no viven como deberían vivir según las normas familiares. Esta discrepancia se ve claramente en uno de los lugares más importantes en mi investigación, un bar cerca del parque más popular de la ciudad. Las latinas en este bar (como en muchos otros bares de la ciudad) no sólo trabajan como meseras, sino que también utilizan este espacio para iniciar relaciones íntimas con clientes de sexo masculino. A cambio de ayuda práctica, ellas les ofrecen servicios sexuales. La ayuda práctica que reciben no sólo consiste en dinero y regalos, sino también en apoyo con 'papeles'. A través de contactos con hombres beliceños ${ }^{9}$, las latinas pueden obtener permiso de trabajo, residencia, incluso 
la nacionalidad beliceña. Además, cuando la policía las encarcela por falta de 'papeles', estos hombres también pueden pagar la suma para liberarlas.

Las relaciones entre las latinas y los clientes normalmente se tratan de una combinación entre cariño y una actitud estratégica (ver van Wijk, 2004, pp. 41-44). La manera en que Chevannes (2001, p. 199) describe este tipo de relaciones es útil:

On the one hand is the state of being in love, on the other prostitution. And in between are various stages of conjugal bonding ... as well as the various types of negotiated sexuality having elements of the transactional, but not quite as cold as prostitution. The difference between one type and the next is neither sharp nor sudden...

Así que dentro de sus relaciones con clientes, las latinas maniobran entre amor y prostitución. Esto es posible en el contexto beliceño porque muchas veces las relaciones entre beliceños y beliceñas tienen una base abiertamente económica (McClaurin, 1996, p. 114). En su estudio de bailarinas y prostitutas en una isla de Belice, van Wijk (2004, p. 43) argumenta que "the continuum between love and money creates a space for female sex workers to blend into a society where relationships in which money plays an important role are not necessarily associated with prostitution".

Lo precedente no significa que las prostitutas en Belice no se ven estigmatizadas (van Wijk, 2004, pp. 44-46). Las latinas que deciden iniciar relaciones íntimas con clientes necesitan tomar en cuenta que esa decisión les hace más vulnerables a la explotación. Pero muchas veces este riesgo lo compensa la ayuda práctica que ellas puedan recibir.

Sin embargo, para que ellas puedan regresar con dignidad, es importante que sus familiares no se den cuenta de este tipo de relaciones. Las latinas saben que sus familiares esperan de ellas que no se vuelvan 'locas', que 'no se metan con hombres'. Mantener una relación con una pareja no causa problemas, pero mantener varias relaciones con diferentes hombres les pone en riesgo de cotilleo dañoso y, en el peor caso, de rechazo. Debido a su trabajo, muchas de ellas tienen que justificar su vida o mentir sobre ella. Cuando son madres, utilizan la imagen de la madre que sufre para cuidar a sus hijos (Nencel, 2001, pp. 222-223). Cuando no son madres, pueden utilizar sus responsabilidades como hija para legitimar su vida. Cuando las latinas utilizan estrategias que hacen posible aliviar la situación de una madre enferma o de pobreza extrema, por ejemplo, los familiares casi no tienen derecho a juzgar su forma de vivir (2001, p. 215). Por último, pueden esconder ciertos aspectos de su vida. Cuando le pregunté a la mesera Catarina (25) si su mamá sabe que ella trabaja en un bar, ella me respondió: "No, mi mamá no sabe donde trabajo. Ella piensa que es en un restaurante. Yo tengo miedo de contarle porque va a la iglesia... Las amigas de mi mamá sí lo saben pero no lo ven como algo malo porque ellas hicieron lo mismo.” Esta cita no sólo ilustra cómo las latinas que trabajan en bares se ven obligadas a mentir y a ocultar detalles de su trabajo, sino que también indica que es una estrategia más común.

Además, incluso las latinas que no trabajan en bares viven sus relaciones íntimas de una manera más libre que en su país de origen. En el contexto caribeño de Belice City, los hombres ven a las latinas como mujeres atractivas por ser mujeres extranjeras. 
$\mathrm{Su}$ encanto tiene diferentes componentes. En primer lugar se trata de su apariencia en comparación con las criollas: el color claro de su piel y su pelo liso. También tiene que ver con su vulnerabilidad como migrantes. En términos de trabajo, tanto su imagen dócil -sobre todo cuando trabajan brindando servicios domésticos o como vendedoras- como su imagen sensual -cuando trabajan como meseras- contribuyen a su encanto. Aunque su posición como mujeres extranjeras les puede poner en riesgo de explotación, también la utilizan a su favor. Belice City les ofrece trabajo, sobre todo en los servicios. Su participación en este mercado laboral se ve facilitada por el sistema político que hace posible el acceso a 'papeles' a través de relaciones íntimas, y por la cultura de Belice, en la que se admira su etnicidad. Con respecto a sus familiares en el país de origen, las latinas hacen uso de la distancia física para poder presentar su vida en Belice como una vida aceptable.

Aparte de esconder ciertos aspectos de su vida, otra estrategia para que sus familiares no se preocupen -y que les estimen como mujeres dignas- es establecer un nuevo hogar con una pareja. La mitad de las latinas en mi investigación vive con un hombre en Belice City. El hecho de que casi todos estos hombres sean beliceños (por nacimiento, nacionalización o residencia) significa que casi todas las latinas que viven con una pareja han podido utilizar esta relación para obtener 'papeles'.

\subsection{Dinámica de género en los nuevos hogares en Belice City}

Sin juzgar si una relación entre una latina y su pareja en Belice City está basada en el amor o no, el hecho de vivir juntos siempre tiene un cariz estratégico, principalmente con respecto a la economía del hogar. Las latinas trabajan para poder invertir en sus propios proyectos y para mandar dinero a su hogar familiar. Cuando viven con un hombre, pueden ahorrar en los gastos de la casa y usar más cantidad para sus metas personales porque es la responsabilidad de la pareja -el hombre con quien la latina está viviendo- encargarse de los gastos del nuevo hogar, como la renta, las cuentas y las compras. La mujer no necesariamente tiene que contribuir a eso. Con Juan (26), un soltero sin trabajo, hablé sobre el trabajo de sus hermanas. Cuando le pregunté si ellas dan su sueldo a sus parejas, me dijo: “¿Que si dan su dinero a su marido? Claro que no. Una mujer no da a su marido. Es mejor al revés: un hombre debe mantener a su mujer." Se podría ver esta cita como una negación de la contribución económica de la mujer a su hogar y como una confirmación de su dependencia. Pero al mismo tiempo, esta opinión ‘tradicional' indica una actitud todavía común que abre ciertos caminos para las latinas.

Las diferentes expectativas que los familiares tienen hacia los hombres y las mujeres generan una dinámica muy particular dentro de los nuevos hogares en Belice City. En las historias de los migrantes se puede detectar que el vínculo entre un hombre y su hogar familiar es menos fuerte que el vínculo entre una mujer y su familia. Aunque se aprecia un hombre que ayuda a su familia, en mi investigación no lo ven como una obligación familiar. Esa actitud tiene que ver con la expectativa general de que el hombre es más autónomo; establecerá su propio hogar y tendrá que invertir en ello (Steenbeek, 1995, p. 73) ${ }^{10}$, lo cual se refleja en los objetivos de los hombres migrantes. Ellos ven el hecho de buscar a una mujer como parte fundamental de su estancia en Belice. Por ejemplo, a Lorenzo (22), un guardia soltero, le pregunté cuál era su meta en Belice y me respondió: "Hacer mi casa y acompañarme." 
Ninguna latina me ha dicho eso. Como me dijo la mesera Catarina (25): "No vine para acompañarme, vine para trabajar.” Las responsabilidades económicas que deben asumir las latinas respecto al hogar familiar no disminuyen cuando ellas se juntan para hacer su nuevo hogar en Belice City.

Así que la continuación del apoyo familiar es posible precisamente por las diferentes expectativas que se depositan en los hombres y en las mujeres. Cuando una latina se junta con un hombre, sea migrante o no, se espera que él se encargue de los gastos de la casa para que ella pueda seguir mandando dinero a su familia. En las palabras de Yasmin (20), una vendedora, se puede ver bien que para el hombre su nuevo hogar tiene prioridad: "Mi novio quiere mandar dinero a su familia pero como ya tiene su esposa [Yasmin], tiene que mantenerme a mí y a mi hijo.” El dinero que la pareja de Yasmin gana es primeramente para su nuevo hogar mientras Yasmin usa el dinero que ella gana para mandarlo al hogar familiar. Por otra parte, el hecho de que las latinas utilizan su responsabilidad familiar para poder apartar más dinero, no quiere decir que se usa todo ese dinero para los gastos del hogar familiar. Dependiendo de las instrucciones de las latinas, sus familias lo guardan o lo invierten para ellas.

Que las relaciones de las latinas con hombres en Belice City no sólo tienen un carácter estratégico sino también temporal, queda reflejado perfectamente en el caso de Jackeline (21). Ya hace ocho años que vive en Belice City. Hace dos años que se juntó con un beliceño y tuvo dos hijos con él. Su relación empezó de una manera difícil: se encontraron en un bar donde ella estaba trabajando como mesera mientras él todavía estaba con una beliceña. Jackeline me contó cómo la ex-mujer de su pareja la insultaba a menudo cuando su esposo escogió a Jackeline. A pesar de los problemas que Jackeline ha vivido para estar con su pareja, ella ve su futuro sin él, en Honduras. Cuando le pregunté qué iba a pasar con su pareja cuando ella regresara a Honduras, me dijo: "¿Mi marido? iQue lo agarre la que tenía!” Aunque en Belice City sus parejas juegan un papel importante para que las latinas puedan perseguir el objetivo de 'salir adelante', esto no quiere decir que estos hombres son parte de su visión de futuro. Es más, la mayoría prefiere disfrutar sola de su independencia económica en el país de origen, lo que esperan lograr con sus ahorros de Belice.

\section{Conclusión}

Lo precedente intenta dar una imagen de la vida de las latinas en Belice City. No pretendo decir que todas las migrantes centroamericanas en Belice vivan y sueñen como las que forman parte de mi investigación, sino que a partir de sus experiencias y perspectivas argumento que el discurso mundial de la migración internacional necesita de un replanteamiento. En primer lugar respecto a la tendencia de ver a los hombres y a las mujeres por separado. El marco teórico gendered geographies of power dirige la atención a la manera en que las latinas están ubicadas en la geometría del poder que es la migración internacional. Facilitadas por procesos económicos y políticos, las latinas buscan 'salir adelante' a través de la organización de sus hogares transnacionales basada en la negación de normas y prácticas 'tradicionales' de género. Sin embargo, el análisis presentado en este artículo indica que analizar solamente este papel de las latinas no explica cómo funciona el proceso migratorio de ellas. Es precisamente la interacción entre hombres y mujeres lo que 
constituye la migración. Las normas y prácticas presentes en los hogares transnacionales dictan una responsabilidad económica constante de las latinas hacia sus hogares familiares. En contraste, se espera una posición más autónoma de los hombres, quienes deben mantener su nuevo hogar donde las latinas dependen de ellos. En la combinación de hogares que resulta, se trata de valores 'tradicionales' de dedicación familiar y dependencia femenina, pero insertos en un nuevo escenario transnacional donde las latinas encuentran el espacio para buscar cómo ‘salir adelante'.

Esto dirige la atención a la segunda manera en que el discurso de la migración necesita de un replanteamiento, que es respecto al énfasis en la vulnerabilidad o el posible empoderamiento de las mujeres migrantes. El análisis indica que se trata de un proceso de búsqueda de una vida mejor en el que las latinas se encuentran en un continuo donde negocian entre posiciones más vulnerables, donde pueden ejercer poca autoridad, y posiciones más fuertes, donde pueden tomar control de su destino. Pese a su posición en las jerarquías de género y etnicidad, entre otras, las latinas tienen acceso a la migración y a 'salir adelante' pero siempre en términos 'tradicionales' y con cierto riesgo de explotación. Esto significa que en algunos aspectos han podido utilizar su agencia pero en otros su agencia se ve limitada. Por lo tanto, para estas latinas, la migración se ve caracterizada tanto por triunfos como por sacrificios en el escenario transnacional que no se pueden simplificar en una simple cuestión de vulnerabilidad o empoderamiento.

\section{Notas}

1 Los beliceños criollos son de origen africano y europeo y generalmente hablan inglés criollo.

2 Así es como los beliceños criollos se refieren a los migrantes centroamericanos.

3 Usaré la palabra latinas para migrantes centroamericanas porque así se refieren a ellas mismas.

4 Todas las citas que aparecen en este texto son de hondureños.

5 Naturalmente, la migración sigue siendo una aventura arriesgada en la que tanto las mujeres como los hombres corren muchos peligros. Pero en comparación con otros destinos, en particular los Estados Unidos, se conoce Belice como un país accesible.

6 Cuando las latinas entran al país, sólo tienen permiso de quedarse un mes y no pueden trabajar. Tienen la posibilidad de renovar este permiso, pero es un proceso costoso y de todas maneras no pueden trabajar legalmente. Una opción popular para conseguir 'papeles' es relacionarse con un beliceño, lo cual explicaré en otra sección.

7 Aunque muchas tienen familia en Belice City, debido a conflictos familiares pocas viven con ellos.

8 El deseo de regresar al país de origen no necesariamente se convierte en realidad. Sin embargo, solamente la idea de querer regresar influye de manera significativa en la vida de las latinas.

9 Estos hombres beliceños pueden ser criollos o latinos y pueden ser beliceños de nacimiento, inmigrantes con residencia beliceña o inmigrantes nacionalizados.

10 Esta actitud autónoma de los hombres se encuentra tanto en inmigrantes como en beliceños.

\section{Referencias bibliográficas}

Arango, J. (2000). Explaining migration: a critical view. International Social Science Journal (165), 283-296.

Bailey, A. J. (2001). Turning Transnational: Notes on the Theorisation of International Migration. International Journal of Population Geography (7), 413-428. 
Carr, E. (2005). Development and the household: Missing the point? GeoJournal (62), 71-83. Central Intelligence Agency. (2009). The World Factbook, Belize. Recuperado el 17 de septiembre de 2009 de https://www.cia.gov/library/publications/the-world-factbook/ geos/bh.html

Chaney, E. M. (1993). Research on Migration and Women in Latin America and the Caribbean. En E. Acosta-Belén \& C. E. Bose (eds.) Researching women in Latin America and the Caribbean, pp. 99-134. Boulder: Westview Press.

Chant, S. (1998). Households, gender and rural-urban migration: reflections on linkages and considerations for policy. Environment and Urbanization 10 (1), 5-21.

Chant, S. (2007). Gender, Generation and Poverty. Exploring the 'Feminisation of Poverty' in Africa, Asia and Latin America. Cheltenham: Edward Elgar.

Chant, S. \& Craske, N. (2003). Gender in Latin America. London: Latin America Bureau.

Chevannes, B. (2001). Learning to be a man: culture, socialization and gender identity in five Caribbean communities. Kingston: University of West Indies.

De Haan, A. (2006). Migration in the Development Studies Literature. Has It Come Out of Its Marginality? Research Paper No. 2006/19. Helsinki, UNU World Institute for Development Economics Research (UNU-WIDER).

De Oliveira, O. (1991). Migration of women, family organisation and labour markets in Mexico. En E. Jelin (ed.) Family, household and gender relations in Latin America, pp. 101-118. London: Kegan Paul International.

Donato, K., Gabaccia, D., Holdaway, J., Manalansan, M. \& Pessar, P. (2006). A Glass Half Full? Gender in Migration Studies. International Migration Review 40 (1), 3-26.

Escrivá, Á. (2000). ¿Empleadas de por vida? Peruanas en el servicio doméstico de Barcelona. En Papers (60), 327-342.

Evergeti, V. \& Zontini, E. (2006). Introduction: Some critical reflections on social capital, migration and transnational families. Ethnic and Racial Studies 29 (6), 1025-1039.

Gardner, K. \& Grillo, R. (2002). Transnational households and ritual: an overview. Global Networks 2 (3), 179-190.

Glick Schiller, N., Basch, L. \& Blanc-Szanton, C. (1992). Transnationalism: A New Analytic Framework for Understanding Migration. En S. Vertovec \& R. Cohen (eds.) (1999) Migration, Diasporas and Transnationalism, pp. 26-49. Cheltenham: Edward Elgar.

González de la Rocha, M. (1994). The resources of poverty. Women and survival in a Mexican city. Cambridge: Blackwell Publishers.

González de la Rocha, M. (2001). From the Resources of Poverty to the Poverty of Resources? The Erosion of a Survival Model. Latin American Perspectives 28 (4), 72-100.

Grieco, E. M. \& Boyd, M. (1998). Women and migration: incorporating gender into international migration theory. Florida State University, College of Social Sciences, Center for the Study of Population (Working Paper).

Hirsch, J. (1999). En el Norte la Mujer Manda. Gender, Generation, and Geography in a Mexican Transnational Community. American Behavioral Scientist 42 (9), 1332-1349. Hondagneu-Sotelo, P.(1994). Gendered Transitions. Mexican Experiences of Immigration. Berkeley: University of California Press.

Jolly, S. \& Reeves, H. (2005). Gender and Migration. Overview Report. Institute of Development Studies. BRIDGE. Recuperado el 10 de junio de 2009 de http://www.bridge. ids.ac.uk/reports/CEP-Mig-OR.pdf

Kivisto, P. (2001). Theorizing transnational immigration: a critical review of current efforts. 
Ethnic and Racial Studies 24 (4), 549-577.

Levitt, P., DeWind, J. \& Vertovec, S. (2003). International Perspectives on Transnational Migration: An Introduction. International Migration Review 37 (3), 565-575.

Mahler, S. \& Pessar, P. (2001). Gendered Geographies of Power: Analyzing Gender across Transnational Spaces. Identies: Global Studies in Culture and Power 7 (4), 441-459.

Mahler, S. \& Pessar, P. (2006). Gender Matters: Ethnographers Bring Gender from the Periphery towards the Core of Migration Studies. International Migration Review 40 (1), 27-63.

Massey, D. (1993). Power-geometry and a progressive sense of place. En J. Bird, B. Curtis, T. Putnam \& G. Robertson (eds.) Mapping the Futures: Local Cultures, Global Change, pp. 59- 69. London: Routledge.

McClaurin, I. (1996). Women of Belize. Gender and Change in Central America. New Brunswick: Rutgers University Press.

Molyneux, M. (2002). Gender and the Silences of Social Capital : Lessons from Latin America. Development and Change 33 (2), 167-188.

Morales Gamboa, A. (2007). La diáspora de la posguerra. Regionalismo de los migrantes y dinámicas territoriales en América Central. Disertación doctoral no publicada, Universiteit Utrecht, Utrecht, Reino de los Países Bajos.

Morales, A. \& Castro, C. (2002). Redes Transfronterizas. Sociedad, Empleo y Migración entre Nicaragua y Costa Rica. San José: FLACSO.

Nencel, L. (2001). Ethnography and Prostitution in Peru. London: Pluto Press.

Organización Internacional para las Migraciones / Sistema de Información Estadística sobre las Migraciones en Centroamérica (2004). Inmigración y emigración en Centroamérica a inicios del siglo XXI: Sus características e impacto. Serie Perfil de los Migrantes No 1. Recuperado el 17 de septiembre de 2009 de http://163.178.140.43/ descargas/documentos/siemca05.pdf

Oso Casas, L. (2008). Migración, género y hogares transnacionales. Recuperado el 24 de septiembre de 2009 de http://dei.ugr.es/doctorado/private/cursos_pdf/Mesa_7 LauraOso.pdf

Posso Quiceno, J. (2007). La migración colombiana hacia España, las redes de hogares transnacionales y los cambios en las relaciones de género. En Amérique Latine Histoire et Mémoire. Les Cahiers ALHIM, 14. Recuperado el 22 de septiembre de 2009 de http://alhim.revues.org/index2162.html

Pribilsky, J. (2004). 'Aprendemos a convivir': conjugal relations, co-parenting, and family life among Ecuadorian transnational migrants in New York City and the Ecuadorian Andes. Global Networks 4 (3), 313-334.

Pessar, P. \& Mahler, S. (2003). Transnational Migration: Bringing Gender In, International Migration Review 37 (3), 812-846.

Portes, A. (1997). Immigration Theory for a New Century: Some Problems and Opportunities. International Migration Review 31 (4), 799-825.

Portes, A., Guarnizo, L. \& Landolt, P. (1999). The study of transnationalism: pitfalls and promise of an emergent research field. Ethnic and Racial Studies 22 (2), 217-237.

Pries, L. (1999). Migration and Transnational Social Spaces. Aldershot: Ashgate.

Ramírez, C., García Domínguez, M. \& Míguez Morais, J. (2005). Crossing Borders: Remittances, Gender and Development. (Working paper) Santo Domingo: INSTRAW.

Ribas, N. (2001). ¿Estrategias transnacionales? Una pregunta acerca de las migraciones 
femeninas en España. Arxius (5), 69-92.

Sassen, S. (2002). Women's Burden: Counter-Geographies of Globalization and the Feminization of Survival. Nordic Journal of International Law 71, 255-274.

Stack, C. B. (1974). All Our Kin. Strategies for Survival in a Black Community. New York: Harper \& Row.

Steenbeek, G. (1995). Vrouwen op de drempel. Gender en moraliteit in een Mexicaanse provinciestad. Amsterdam: Thela Publishers.

Stephen, L. (1993). Antropological Research on Latin American Women: Past Trends and New Directions for the 1990s. En E. Acosta-Belén \& C. E. Bose (eds.) Researching women in Latin America and the Caribbean, pp. 99-134. Boulder: Westview Press.

Tamagno, C. (2003). Entre acá y allá. Vidas Transnacionales y Desarrollo. Peruanos entre Italia y Perú. Disertación Doctoral no publicada, Wageningen Universiteit, Wageningen, Reino de los Países Bajos.

Van Wijk, J. (2004). Sun, sea and sharks. Male and female prostitution on Ambergris Caye, Belize. LOVA: Tijdschrift voor de Feministische Antropologie, 25e jaargang, no. 2, $32-48,41-44$.

Vertovec, S. (2004). Trends and Impacts of Migrant Transnationalism. Working Paper 3. Oxford: University of Oxford (Centre on Migration, Policy and Society).

Waldinger, R. \& Fitzgerald, D. (2004). Transnationalism in Question. The American Journal of Sociology 109 (5), 1177-1195.

Zoomers, E. B. (2006). Op zoek naar eldorado. Over internationale migratie, sociale mobiliteit en ontwikkeling. Nijmegen: discurso Radboud Universiteit Nijmegen. 